\begin{tabular}{|l|l|} 
REVISTA Revista Educación \\
ISSN: 0379-7082 \\
ISSN: 2215-2644 \\
revedu@gmail.com \\
Universidad de Costa Rica \\
Costa Rica
\end{tabular}

\title{
Formación de docentes en servicio de Educación Secundaria en México
}

Rivera Morales, Alicia; Alfageme-González, M. Begoña

Formación de docentes en servicio de Educación Secundaria en México

Revista Educación, vol. 43, núm. 1, 2019

Universidad de Costa Rica, Costa Rica

Disponible en: http://www.redalyc.org/articulo.oa?id=44057415003

DOI: https://doi.org/10.15517/revedu.v43i1.28446

Esta obra está bajo una Licencia Creative Commons Atribución-NoComercial-SinDerivar 3.0 Internacional. 


\section{Formación de docentes en servicio de Educación Secundaria en México}

\section{Teacher Training for In- Service Secondary School Teachers in Mexico}

Alicia Rivera Morales

Universidad Pedagógica Nacional, México

arivera@upn.mx

\author{
M. Begoña Alfageme-González \\ Universidad de Murcia, España \\ alfageme@um.es \\ (DD http://orcid.org/0000-0003-4148-7620
}

DOI: https://doi.org/10.15517/revedu.v43i1.28446

Redalyc: http://www.redalyc.org/articulo.oa?id=44057415003
Recepción: 03 Abril 2017

Aprobación: 05 Noviembre 2018

\section{RESUMEN:}

La formación docente viene condicionada por las políticas educativas de cada país, así como la consideración que de dicha profesión tiene la sociedad. Así, las características de los docentes y su formación son uno de los elementos clave para la educación, para la mejora de la práctica educativa y para su desarrollo profesional. Se refleja aquí un estudio para conocer la formación que tienen los docentes en servicio en cuatro estados mexicanos. La muestra consistió en 600 docentes de 43 escuelas con distinto nivel de logro (alto y bajo), realizándose la recogida de información mediante encuesta. Los docentes mexicanos se actualizan en su formación, fundamentalmente en temas disciplinares, si bien consideran que la mayor incidencia en su formación la tienen los contenidos más cercanos a lo didáctico-pedagógico.

Palabras ClaVe: Competencias docentes, desarrollo de la educación, enseñanza secundaria, formación profesional.

\section{ABSTRACT:}

Teacher training is conditioned by the educational policies of each country, as well as the consideration society has of such profession. Thus, teacher characteristics and their training are one of the key education elements for the improvement of educational practices and teacher professional development. This study was conducted with the aim of knowing about the training in-service teachers have in four Mexican states. The sample consisted of 600 teachers from 43 schools with different level of achievement (high and low). A survey was used to collect information. Mexican teachers basically update their disciplinary knowledge; although they consider that the greater incidence in their formation is more related to didactic-pedagogical contents. KEYWORDS: Teacher competencies, educational development, secondary education, professional formation.

\section{INTRODUCCIÓN}

Las políticas de formación docente en los gobiernos nacionales o locales son una necesidad (Archibald, Coggshall, Croft y Goe, 2011; European Comissión, 2012; MECD, 2014; Wei, Darling Hammond, Andree, Richardson y Orphanos, 2009). La bibliografía consultada destaca el requerimiento de una conexión entre el desarrollo profesional y los aprendizajes docentes, en el sentido señalado por Easton (2008), pero también con la experiencia del alumno y sus aprendizajes (Bolívar, 2005; Barber y Mourshed, 2007; Borko, 2004; Cochram-Smith, 2005; Escudero, 2011; Escudero, González y Rodríguez, 2013; European Commission, 2012; Pérez Gómez, 2010; Wei y otros, 2009). Bolívar (2005) indica que se encuentran evidencias de cómo un profesorado bien cualificado influye en el aprendizaje de los estudiantes, pero no de cómo las políticas educativas contribuyen a capacitar a los profesores y a mejorar los niveles de consecución de los alumnos. Así, uno de los retos para la mejora de la educación es aumentar la formación de los docentes (Barber y Mourshed, 2007; MECD, 2014; OCDE, 2005; Sanz, Hernando y Mula, 2015) desarrollando un mayor y más profundo saber teórico, técnico y ético (Naval, 2013, p. 125). 
En México, las políticas educativas que conforman la profesión docente, afectan sin duda a la formación y a su consideración. Según Ramírez-Rosales (2010):

La profesión magisterial en México se encuentra en crisis, los maestros son el centro de las críticas del deterioro del sistema educativo, se dice que son los culpables de los deficientes resultados evaluatorios de los alumnos y de los suyos propios. (p. 100)

En este sentido, se empeora la formación docente cuando se les pide a los maestros que para formarse paguen ellos o que para actualizarse en su docencia compren ellos sus propios materiales docentes, a la vez que socialmente el perfil de maestro está "sustentado en atributos más profesionales: competencias en el manejo e implementación de la tecnología en sus clases, conocimiento experto de los contenidos escolares, formas de enseñanza innovadoras" (Ramírez-Rosales, 2010, p. 105).

La Reforma Educativa del presente sexenio en México ha generado un efecto singular en la educación, en la escuela y especialmente en los docentes, eje de la reforma, a quienes llegan directamente los cambios. Además, puesto que los docentes son uno de los actores principales que pueden participar para mejorar la práctica de la evaluación e incidir en la calidad de la educación, son a veces también considerados como los únicos responsables de los resultados de dicha calidad. De ahí, la relación establecida por el discurso oficial entre la calidad educativa y la formación de los maestros nos lleva a pensar que al evaluarlos y capacitarlos se conseguirá mejores resultados. Situación que ha propiciado un momento coyuntural y de interés por recuperar en esta investigación información valiosa que permita interpretar quiénes son los docentes que viven la actual política educativa, así como su formación inicial y continuada.

No en vano, esta línea de trabajo ha sido una de las destacadas en la reunión nacional sobre Ciencias Sociales que el grupo de trabajo de Educación, promovido por el Consejo Consultivo de Ciencias de la Presidencia de la República, el Consejo Nacional de Ciencias y Tecnología (CONACYT) y la Academia Mexicana de las Ciencias, celebrada en octubre de 2015, con el título "Hacia dónde va la ciencia en México". En dicha reunión se señalan investigaciones prioritarias que se deben realizar para mejorar la calidad de la educación en México, entre ellas "la formación en servicio y desarrollo profesional también es clave para desarrollar modalidades más adecuadas que los tradicionales cursos de actualización cuya eficacia se ha mostrado limitada" (FloresCrespo y otros, 2016, p. 1300).

Se debe entender, entre otros aspectos, que los saberes y las competencias docentes son resultado de la trayectoria de formación profesional, pero también de los aprendizajes adquiridos a lo largo de la vida, dentro y fuera de la escuela y en el ejercicio mismo del quehacer docente y de su propio tiempo libre. Por tanto, centramos nuestro análisis en la formación docente como ayuda al desarrollo profesional, intentando conocer cuál es la formación que realizaron en los últimos cinco años, como mejora de la calidad de su docencia. Nuestro interés por tanto está centrado en conocer qué formación tienen los docentes en ejercicio en cuatro estados mexicanos.

En este sentido se analiza la formación de los docentes teniendo en cuenta su contenido, centrándonos en los contenidos del conocimiento profesional definidos por Shulman (2005) considerados como los contenidos más importantes del conocimiento profesional de base para la enseñanza y que se pueden ver más ampliamente explicados en el trabajo de Escudero, González y Rodríguez (en prensa). Dichos contenidos del conocimiento profesional se pueden resumir en los siguientes conocimientos: 1) Contenidos disciplinares: estructuras sintácticas y sustantivas del conocimiento disciplinar; 2) Pedagógico general: grandes principios y estrategias docentes que trascienden la enseñanza de las materias; 3) Curricular: programas, materiales y otras recursos para la enseñanza; 4) Pedagógico/didáctico: transformaciones del contenido disciplinar para llevar a cabo la preparación de la enseñanza y facilitar el aprendizaje; 5) De los estudiantes: conocimientos previos, estilos de aprendizaje y otras características; 6) De los contextos educativos: trabajo en grupo, gobierno y cultura del medio escolar; 7) Finalidades, valores educativos, bases filosóficas e históricas; y 8) Razonamiento pedagógico: interpretación de los contenidos, desarrollo de materiales, reflexión y creación en la práctica. 


\section{MÉTODo}

Este trabajo forma parte del estudio Uso de los resultados de evaluación en procesos de mejora en educación secundaria, desde la perspectiva de los actores, llevado a cabo en cuatro entidades de la República Mexicana (Ciudad de México, Durango, Oaxaca y Sonora), elegidas para comparar sus contextos preestablecidos y contrastados por su nivel de logro educativo conocido a priori. Para el caso de Sonora, Durango y Ciudad de México fueron seleccionadas las escuelas concentradas en la base de datos de la Secretaría de Educación Pública (SEP), de acuerdo con los puntajes obtenidos en la Evaluación Nacional del Logro Académico en Centros Escolares (ENLACE). Las escuelas de Oaxaca fueron seleccionadas por las autoridades educativas estatales, quienes definieron las de mayor y menor nivel de logro académico. En cada entidad fueron seleccionadas 10 escuelas secundarias públicas: cinco de alto logro y cinco de bajo logro. En la Ciudad de México se incorporaron tres escuelas particulares. En total fueron consideradas 43 escuelas secundarias.

Para dar respuesta al estudio planteado en la investigación se diseñó y aplicó un cuestionario mixto (con preguntas cerradas y abiertas) para conocer las trayectorias profesionales y laborales de los docentes. Se solicitó a los directivos la aplicación del instrumento a todos los profesores de las 43 escuelas, de los cuales recogimos aproximadamente el $60 \%$, lo que representa un total de 600 profesores de educación secundaria encuestados. La mayoría son de Ciudad de México (29,7\%), en congruencia a que en el centro del país se concentra la mayor cantidad de población que en cualquier otra entidad federativa; 26,9\% de Oaxaca; 22,9\% de Durango y el $20,5 \%$ de Sonora. Estos datos reflejan al mismo tiempo el número de docentes en relación con el número de habitantes de cada entidad federativa y en consecuencia del número de alumnos, pero implícitamente muestra el porcentaje de aceptación de los profesores para dialogar sobre su historia profesional y personal.

Se presentan aquí los datos que hacen referencia a uno de los objetivos del estudio, centrado en indagar acerca de los trayectos de formación inicial y continua de los profesores de las 43 escuelas, más concretamente como ya se ha señalado en la introducción de este trabajo, se pretende conocer cuál es la formación que realizaron los docentes en los últimos cinco años.

A continuación, se presentan datos que caracterizan nuestra muestra en función del género y la edad de los docentes, así como la modalidad y el turno en el cual laboran para, posteriormente, destacar resultados centrados en la formación inicial y profesionalización de los docentes, así como la formación continua o actualización. Es importante precisar que los hallazgos aquí presentados emergieron de un estudio que se llevó a cabo justo en el inicio de la Reforma Educativa en México, de ahí su relevancia.

\section{Resultados}

En relación con el género, las encuestas indican que en su mayoría los docentes participantes son 56\% del género femenino y $44 \%$ del masculino. El ejercicio de la docencia está siendo desarrollado por mujeres, género que predomina en México, y que acceden a una formación que les permite desempeñar un trabajo con incidencia en el desarrollo de adolescentes.

En cuanto a la edad de los docentes entrevistados, el 35\% son mayores de 51 años; el 29\% tiene entre 31 y 40 años; 26\% de 41 a 50; y un 10\% es menor a los 30 años de edad. Estos datos dan cuenta que la mayoría de los profesores de este estudio tiene amplia experiencia en el campo educativo, pero también de que la población docente está formada por población de cada vez más edad, puesto que es un hecho que la mayoría de la muestra tiene más de 30 años. Se observa, por tanto, que los docentes de secundaria han pasado un largo tramo de su vida en las aulas escolares. Además, los docentes de mayor edad se ubican en Oaxaca, en tanto en las otras tres entidades el promedio de edad se distribuye equitativamente.

La mayoría de docentes del estudio (73\%) pertenece a escuelas que su desempeño se ubica en alto nivel de logro, es decir, su rendimiento es mayor en relación con las expectativas que se tiene de las escuelas, mientras que el $27 \%$ de docentes pertenece a escuelas de bajo logro o de tipo B, es decir, escuelas que 
con condiciones favorables, su rendimiento es menor frente a las expectativas que hay sobre ellas. Las mujeres se ubican en mejor desempeño académico no solo porque son mayoría en el estudio, sino por su dedicación y responsabilidad histórica de educar a las jóvenes generaciones, y por su constante participación y autoformación en su profesión.

Respecto de la modalidad de las escuelas, la mayor parte de los docentes trabaja en escuelas secundarias de la modalidad general (58\%), de acuerdo con la realidad puesto que son estas escuelas las que tienen un mayor porcentaje de profesores, son escuelas que poseen una larga historia en la formación de la educación secundaria en México, se crean antes que las otras modalidades y prevalecen después de su creación; el $32 \%$ en técnicas y en menor porcentaje en particulares (4\%), telesecundarias (3\%) y para trabajadores (3\%). Tanto en las escuelas de alto como en las de bajo logro, los docentes se ubican principalmente en la modalidad de general, seguidas de la modalidad técnica, como se puede ver en la Figura 1.

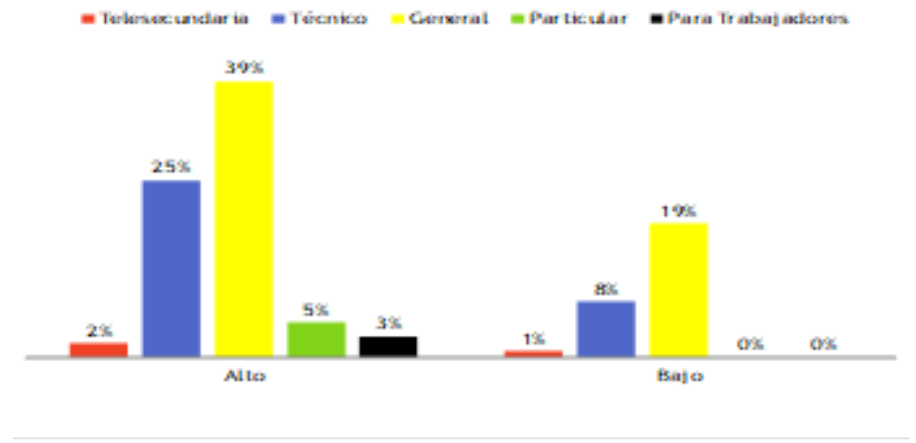

FIGURA 1

Figura 1. Docentes inscritos en escuelas de alto y bajo logro

La mayoría de los profesores de este estudio trabaja en el turno matutino (77\%), el $8 \%$ en turno vespertino (en los estados de Durango, Oaxaca y Sonora), el 4\% en el turno nocturno (en la Ciudad de México) y el 11\% en la Ciudad de México dicen trabajar a la vez en los turnos matutino y nocturno. Esto muestra la preferencia y el reconocimiento hacia las escuelas del turno matutino, es una primera opción, un deseo de acudir y ser aceptado en dicho turno por los profesores.

\section{Formación inicial y profesionalización}

Al estudiar la formación de los docentes en ejercicio podemos ver que el $61 \%$ de los docentes tiene el grado de licenciatura, que es uno de los requisitos para ejercer la docencia en dicho nivel. El 22\% de docentes ha obtenido el grado de maestría, el $13 \%$ de docentes posee una especialidad afín al curso que imparte y en menor porcentaje $4 \%$ tiene un doctorado. Se indica en la Figura 2, el último grado de estudios por área disciplinaria de los docentes. Como podemos ver, en su mayoría (24\%) de los docentes tiene licenciatura en Ciencias Sociales, el 18\% en Humanidades y Artes, el 13\% en Físico Matemáticas y el 6\% en Biológicas y de la Salud, situación que refleja el porcentaje de tiempo de las asignaturas que se cursan en el plan de estudios de la educación secundaria. Si bien son los del área de Humanidades y Artes los que tienen más estudios superiores, puesto que representan el mayor porcentaje en la especialidad, maestría y posgrado (doctorado). Los datos indican que los docentes con nombramiento definitivo o de base son los que aumentan sus estudios con especialidades, maestría o posgrado, pero no los interinos o docentes con otros nombramientos. 


$$
\begin{aligned}
& \text { Ciencias Sociales } \quad \text { Físico Matemáticas } \\
& \text { Biológicas y de la Salud }=\text { Humanidades y de Artes }
\end{aligned}
$$

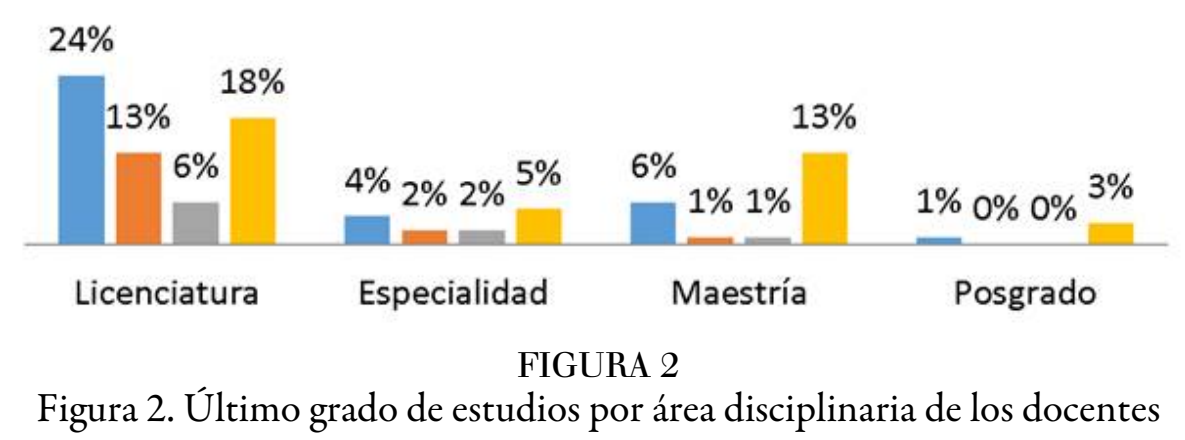

\section{Formación continua o actualización}

Cuando se les pregunta a los docentes cuántos cursos de formación o preparación continua han realizado en los últimos cinco años, la respuesta se recoge en la Figura 3.

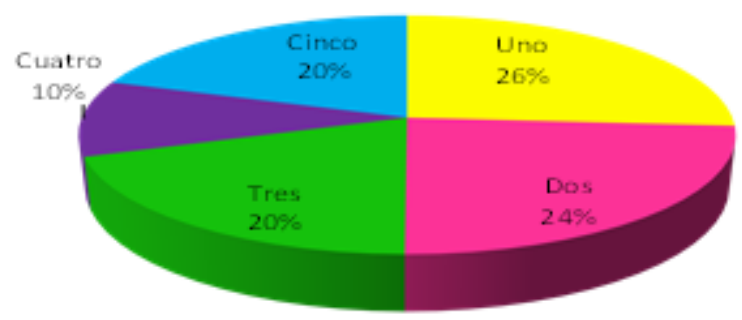

FIGURA 3

Figura 3. Total de cursos tomados en los últimos cinco años por los docentes

Los docentes que tienen solamente licenciatura son quienes acuden más a cursos, seguidos por los de maestría, especialidad y por último los de doctorado (Figura 4). El que a mayor grado académico haya una menos asistencia a cursos de actualización puede indicar que se consideran más formados o que los propios estudios para mejorar la titulación facilitan esta formación. Asimismo, los docentes de las escuelas de alto logro acuden más a cursos de formación que los de escuelas de bajo logro (Figura 5). 


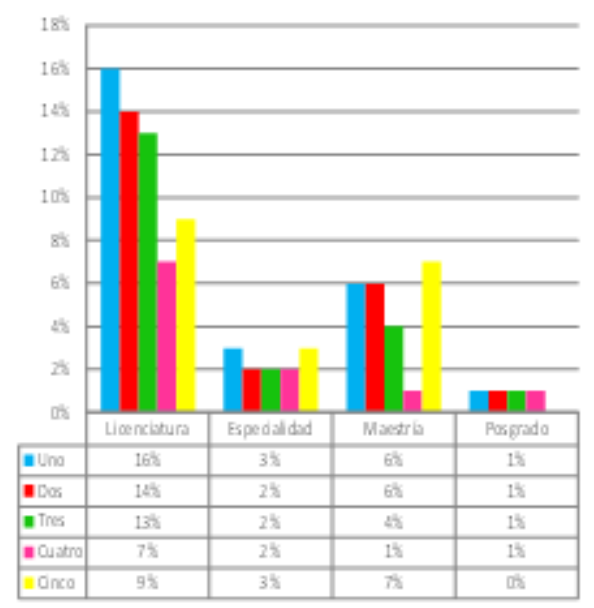

\section{FIGURA 4}

Figura 4. Último Grado de estudios- Total de cursos tomados en los últimos cinco años por los docentes

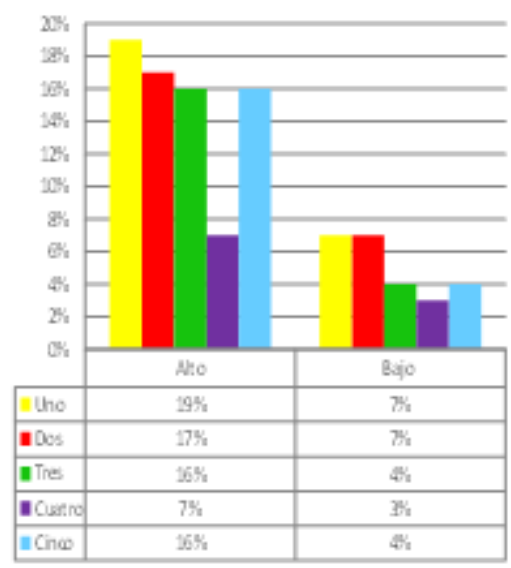

FIGURA 5

Figura 5. Total de cursos tomados por los docentes- Logro académico de la escuela 


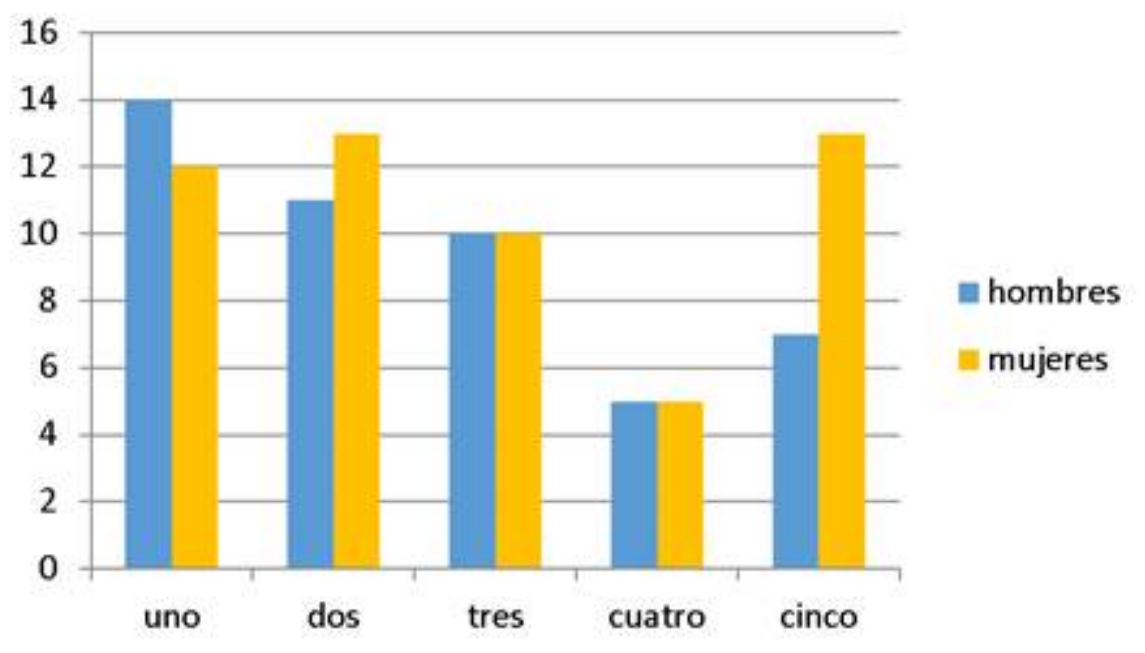

FIGURA 6

Figura 6. Cursos realizados según género

Como se detalla en la Figura 6, en su conjunto son los docentes de alto logro quienes mantienen los más altos porcentajes en acudir a cursos de actualización, así como quienes laboran en la modalidad de educación general, seguidos en este caso por los de la modalidad de técnica, en tercer lugar el profesorado que labora en escuelas particulares y finalmente los de telesecundaria. Seguramente porque son los de la modalidad general las mujeres docentes quienes parecen realizar más formación que los hombres.

Se les solicitó a los encuestados que señalaran, por orden de prioridad, cuatro cursos de formación que hubieran realizado en los últimos cinco años. Así, los cursos que marcaron en la opción uno como más prioritarios pertenecen a las áreas temáticas que se indican en la Figura 7, entre los que, como se puede advertir, es en evaluación donde se han formado la mayoría de los docentes $(20 \%)$, seguidas casi con el mismo porcentaje de las especialidades de humanidades (19\%) y en tercer lugar desarrollo humano (14\%). Además, indicaron que el $97 \%$ de estos cursos corresponden a los ofertados por la Secretaria de Educación Pública (SEP), situación que responde a la obligatoriedad de la oferta y a que permite un mayor acceso al profesorado.

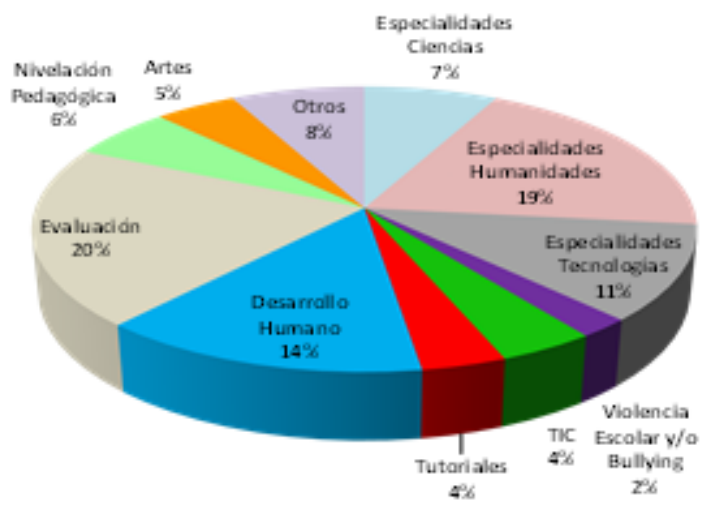

FIGURA 7

Figura 7. Área del curso realizado que los docentes consideran más importante

Profundizando en dicha información, los datos globales nos indican, como aparece en la Figura 8, que las temáticas abordadas en los cursos de formación realizados por los docentes siguen la misma tendencia, siendo los más realizados por los docentes los cursos de evaluación (un total de 18,9\%), seguidos de los relacionados 
con el desarrollo humano (total 14,7\%) y las humanidades (total 14,3\%). Mientras que no rondan un 3\% los cursos menos realizados sobre tutoriales o artes. También se puede ver en la Figura 9 el comportamiento de las temáticas de los cursos por entidad federativa.

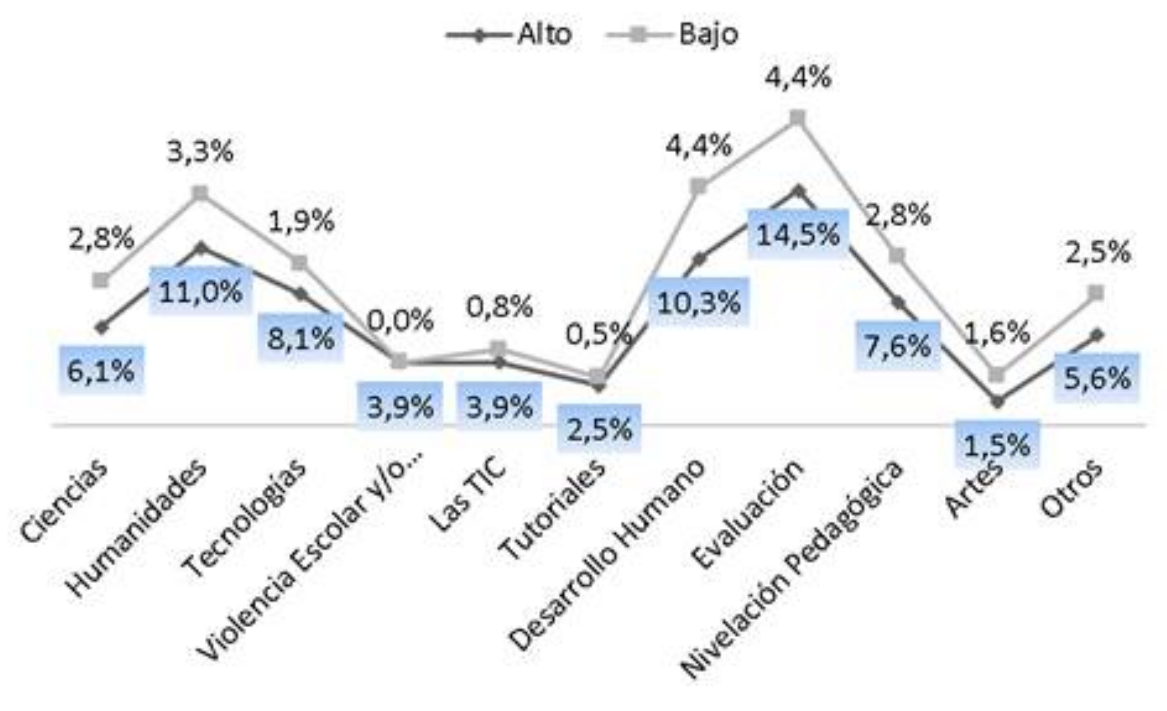

FIGURA 8

Figura 8. Temática del curso de formación por logro académico de las escuelas

$\rightarrow$ Ciudad de Mexico -Durango $\rightarrow$-Oaxaca $\quad *$-Sonora

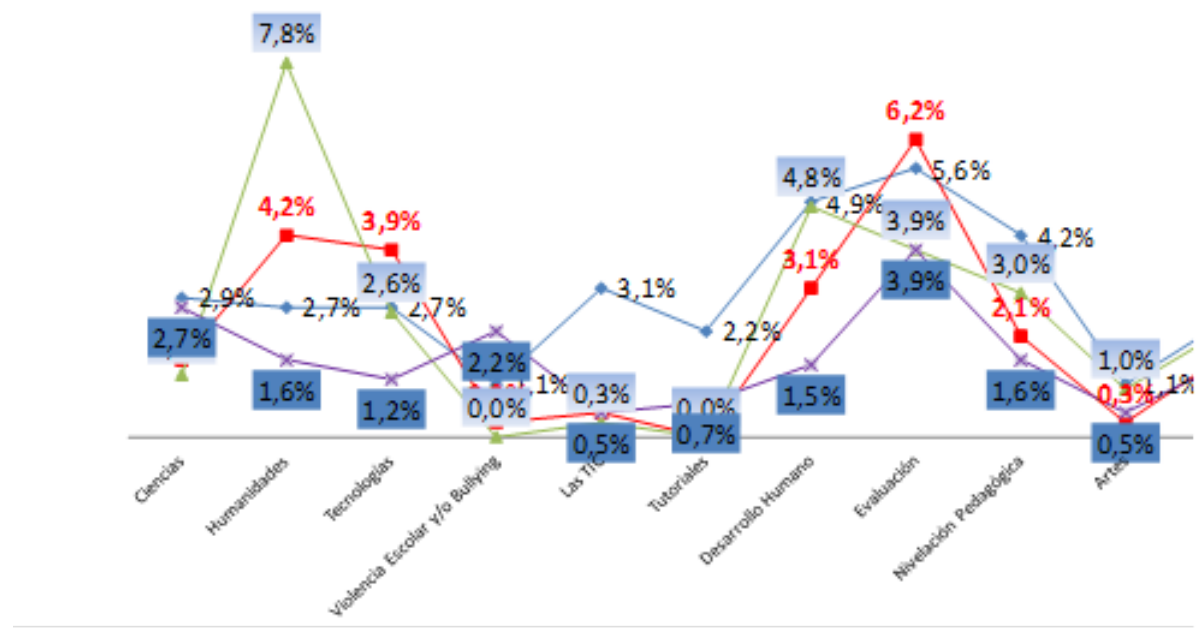

FIGURA 9

Figura 9. Temática del curso de formación por entidad federativa.

Los datos muestran que los docentes están más interesados en conocer acerca del desarrollo humano y la evaluación, podemos inferir que dicho interés se debe a los problemas de los estudiantes de secundaria a los que se enfrentan y a las demandas en procesos evaluativos por parte del Sistema Educativo. Sin embargo, en los temas del curso de formación que los encuestados señalaron como más importantes y con más incidencia para ellos, señalados en la Figura 10, predominan cursos sobre "Mejoras en la enseñanza y competencias", 
"Uso de las TIC", "Relaciones humanas" y "Nivelación pedagógica”. En la Figura 11 también se observan diferencias en el impacto de los cursos según la entidad federativa a la que pertenecen los docentes.

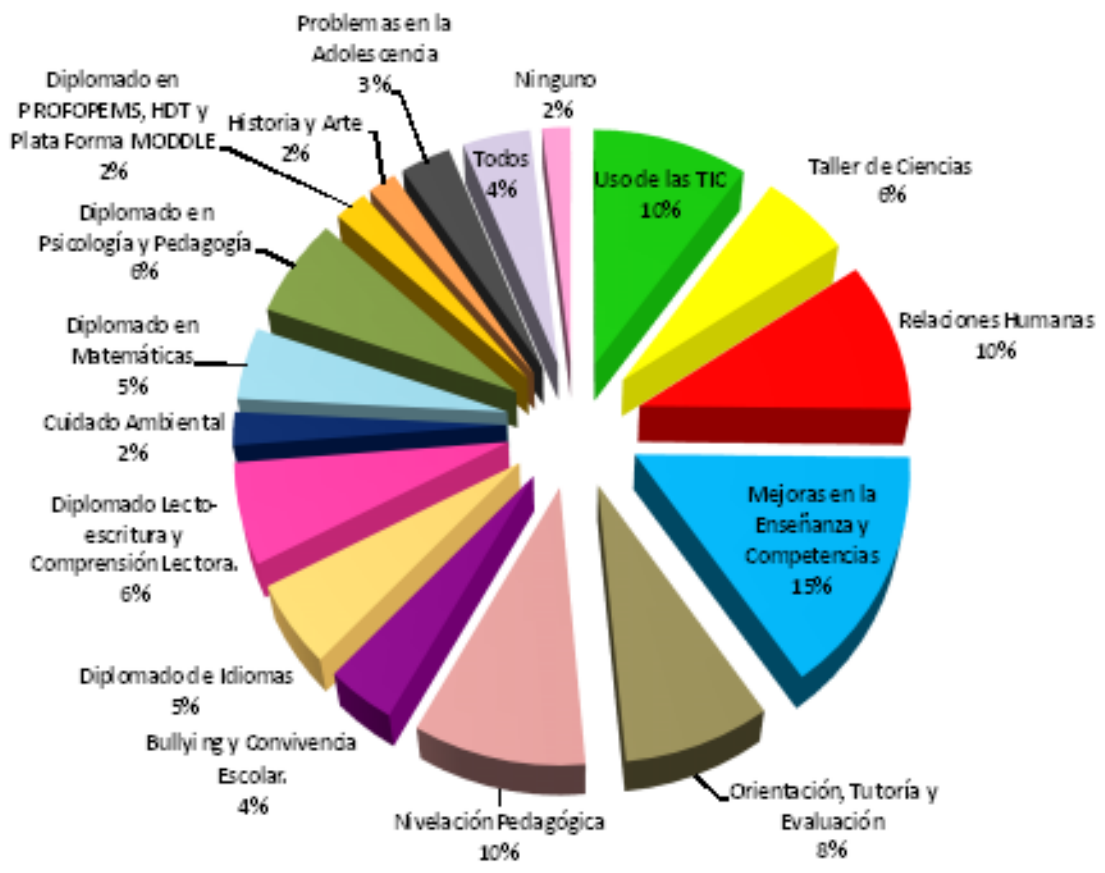

FIGURA 10

Figura 10. Curso que más ha impactado la práctica docente

$$
\rightarrow \text { D.F. -Durango \#-Oaxaca }- \text { - Sonora }
$$

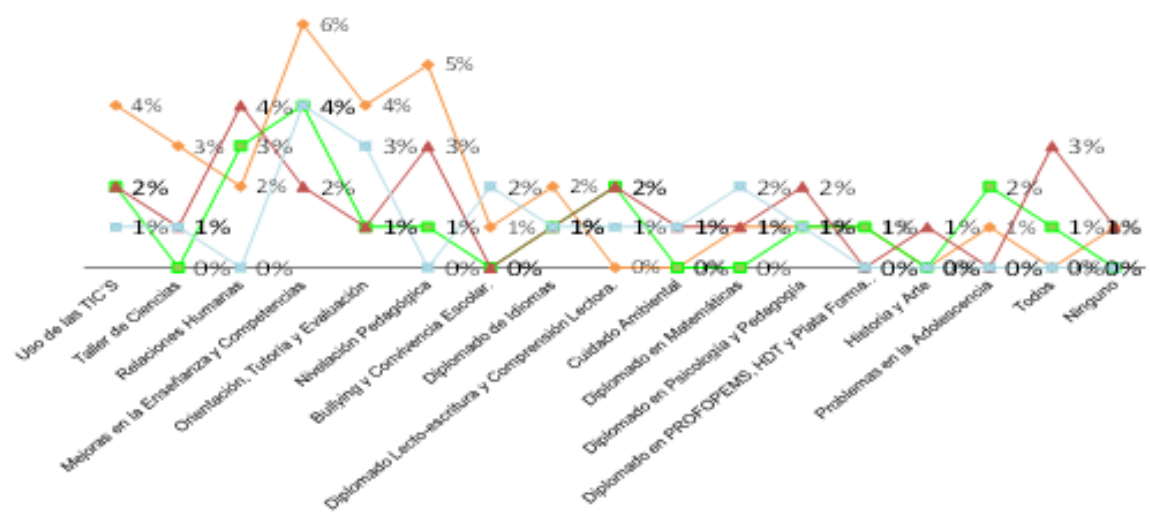

FIGURA 11

Figura 11. Curso que más ha impactado la práctica docente por entidad federativa.

En la Figura 12 se observa que el curso de más impacto para los docentes de todas los tipos de escuelas sigue siendo el de "Mejoras en la enseñanza y competencias", aunque en diferente grado. El mismo dato se corrobora en la Figura 13 donde se ven los cursos que más han impactado a hombres y mujeres, destacándose un mayor impacto en mujeres. En este caso se detalla con más claridad que también los "Usos de las TIC” y los de "Nivelación pedagógica" son cursos que han tenido impacto en los docentes. 


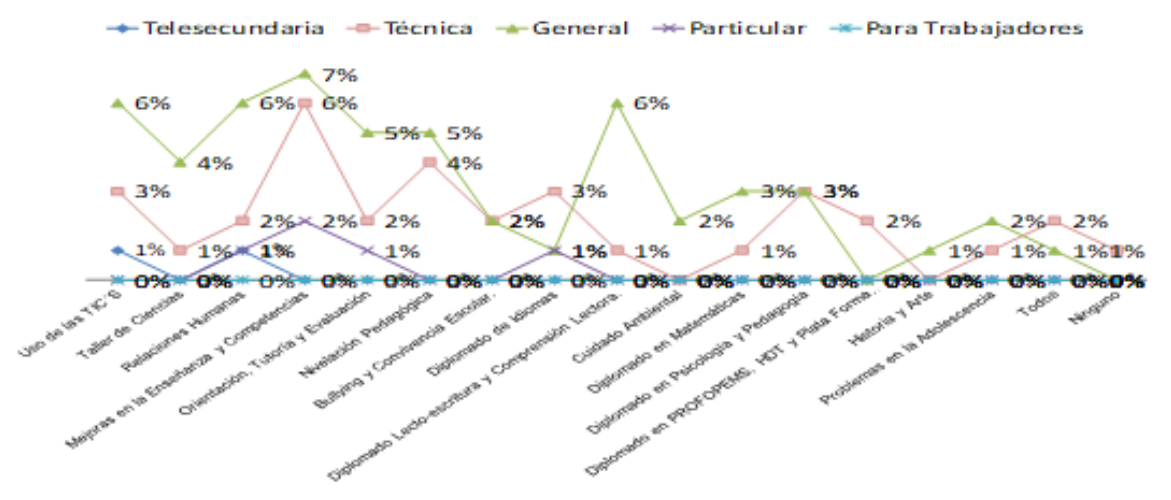

FIGURA 12

Figura 12. Curso que más ha impactado la práctica de los docentes por tipo de escuela.

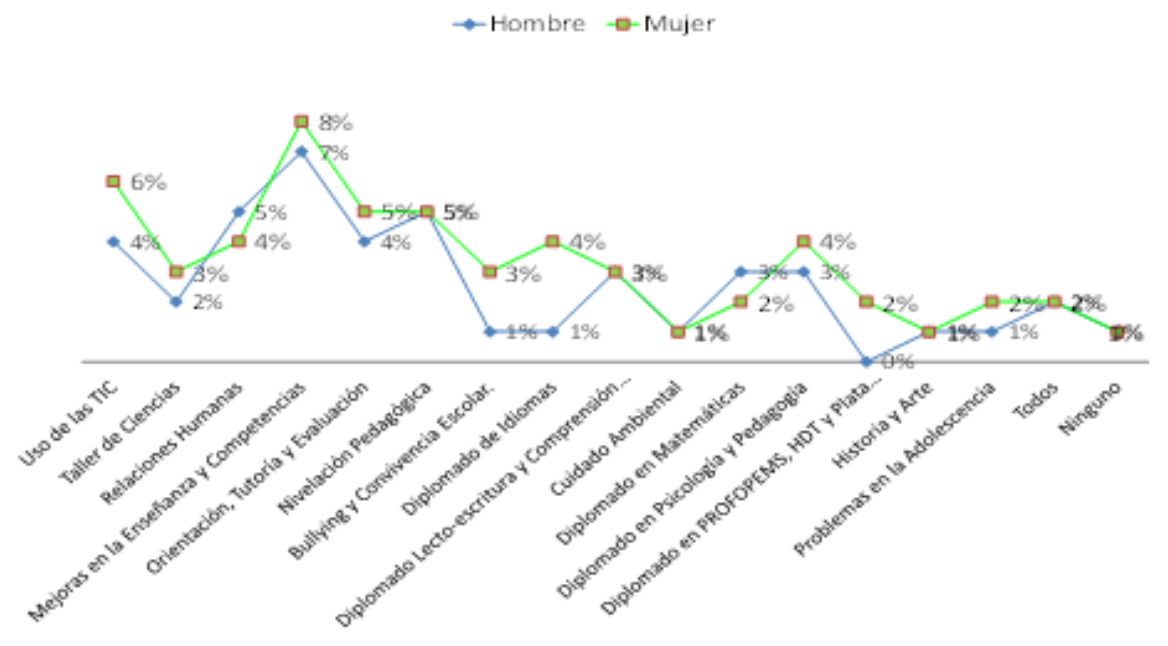

FIGURA 13

Figura 13. Curso que más ha impactado la práctica de los docentes por género

Si bien en la Figura 13 se analiza que son las mujeres quienes tienen mayor asistencia a cursos, esta recurrencia puede deberse a que la docencia en secundaria está sostenida por mujeres en su mayoría. En cualquier caso, la temática en ambos géneros más señalada continúa siendo la de "Mejoras en la enseñanza y competencias".

En los procesos de actualización aún predomina la modalidad presencial, como se puede ver en la Figura 14, en tanto que la semipresencial está en segundo lugar, y en esta era de la conectividad en línea o a distancia aún no es la más demandada por los docentes. Sin embargo, somos conscientes que este dato habrá cambiado en los últimos dos años, dado que las instituciones capacitadoras están comenzando de forma muy activa a ofertar sus cursos y diplomados en línea. 


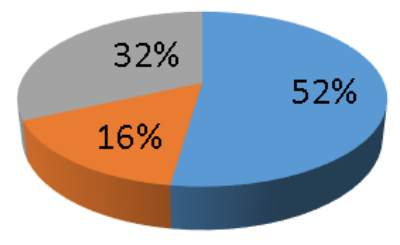

\author{
Presencial \\ En Línea \\ Semi-presencial
}

FIGURA 14

Figura 14. Modalidad del curso señalado como más importante por los docentes

\title{
CONCLUSIONES
}

El trabajo presentado refleja la opinión de 600 docentes de 43 escuelas de cuatro estados de México. Todo el profesorado encuestado ha participado en actividades de formación en los últimos cinco años, lo que supone un porcentaje mayor que otras investigaciones, donde se sitúan en el 91\% de los docentes de Canarias (España) (Alfageme, Arencibia y Guarro, 2016), o en el 94,9\% de la muestra en la investigación española realizada por Alfageme y Nieto (en prensa). Y muy superiores al dato recogido en TALIS 2013 (MECD, 2014), donde se señala un porcentaje de alrededor del $87 \%$, en referencia a las actividades formativas realizadas en los 12 meses anteriores a la encuesta.

Teniendo en cuenta tanto las temáticas de los cursos, como el curso que más incidencia ha tenido en los docentes y, de acuerdo con las categorías definidas por Shulman (2005) y ampliadas con las investigaciones posteriores reflejadas en el trabajo de Escudero y otros (en prensa), predominan los contenidos centrados en el "conocimiento de los contenidos disciplinares" seguidos en orden de mayor a menor importancia por los de "conocimiento pedagógico general", "pedagógico/didáctico" y finalmente el "curricular". Sin embargo, los docentes de la investigación, apuntan como el contenido que más incidencia ha tenido en los docentes uno de conocimiento pedagógico general y señalan, como importantes cursos de desarrollo humano, si bien, este último, con poca incidencia en su labor académica. Sobresale en este estudio que el profesorado de escuelas consideradas de alto logro se preocupa más por su formación continua, como acción importante que influye en su práctica docente.

Los hallazgos aquí presentados llevan a afirmar que los docentes mexicanos están en permanente actualización en temas que para ellos son importantes y en aquellos que saben que tendrán un impacto en su práctica educativa, de ahí que destaquen los contenidos centrados en el "qué", en lo disciplinar, como contenidos de los cursos realizados de formación, aunque la mayor incidencia se centra en los contenidos sobre el "cómo". Esto coincide con la investigación realizada por Escudero y otros (en prensa) en el contexto español, donde la mayor incidencia de la formación se refleja más centrada en los cómo, que en los qué, por qué y para qué.

\section{ReFERENCIAS}

Alfageme-González, M.B., Arencibia, J.S. y Guarro, A. (2016). Contenidos e incidencia en la práctica de la formación docente en Canarias. Profesorado, 20(2), 40-54.

Alfageme-González, M.B. y Nieto Cano, J.M. (en prensa). Los docentes de la Enseñanza Obligatoria en España y las actividades de formación continuada. Perfiles educativos, 1-25.

Archibald, S., Coggshall, J.G., Croft, A. y Goe, L. (2011). High Quality Professional Teacher Development for All Teachers: Effective Allocating Resources. National Comprehensive Center for Teacher Quality. Recuperado de: http://www.gtlcenter.org/sites/default/files/docs/HighQualityProfessionalDevelopment.pdf 
Barber, M.y Mourshed, M. (2007). How the World's Best Performing School Systems Come out on Top. Recuperado de: http://mckinseyonsociety.com/how-the-worlds-most-improved-school-systems-keep-getting-better/

Bolívar, A. (2005). ¿Dónde situar los esfuerzos de mejora?: Política educativa escuela y aula. Educaçao \& Sociedade, 26(92), 859-888.

Borko, H. (2004). Professional development and teacher learning: mapping the terrain. Educational Researcher, 33(8), 3-15.

Cochran-Smith, M. (2005). Teacher education and the outcomes trap. Journal of Teacher Education, 56(5), 411-417.

Easton, L.B. (2008). From professional development to professional learning. Phi Delta Kappan, 89(10), 755-776.

Escudero, J.M. (2011). La formación continuada del profesorado, un tema crucial para la mejora de la educación. Ministerio de Educación, Madrid.

Escudero, J.M., González, M.T. y Rodríguez, M.J. (2013). La mejora equitativa de la educación y la formación del profesorado. Multidisciplinary Journal of Educational Research, 3(3), 206-234.

Escudero, J.M., González, M.T. y Rodríguez, M.J. (en prensa). Los contenidos de la formación continuada del profesorado: ¿qué docentes se están formando? Educación XXI, 1-22.

European Commission (2012). Supporting the Teaching Professions for Better Learning Outcomes. Commission Staff Working Document. Strasbourg.

Flores-Crespo, P. (Coord.), Blanco, E., Cárdenas, S., Cordero Arroyo, G., Díaz-Barriga, F., Jiménez, Y., Martínez Rizo, F. y Ornelas, C. (2016). ¿Por qué no mejora la calidad de la educación básica? RMIE (Revista Mexicana de Investigación Educativa), 71(XXI), 1295-1303.

MECD (Ministerio de Educación cultura y Deporte) (2014). TALIS 2013. Estudio internacional de la enseñanza y el aprendizaje (Informe Español), Madrid: España. MECD. Recuperado de: http://www.mecd.gob.es/dctm/inee/ internacional/talis2013/talispublicacionessep2014.pdf?documentId=0901e72b81adaba0

Naval, C. (2013). Prestigio, autoridad y profesionalización. En V. Pérez-Díaz (Dir.), El prestigio de la profesión docente en España: Percepción y realidad (pp. 125-128). Madrid: Fundación Europea Sociedad y Educación.

OCDE (2005). Teachers matter: attracting, developing and retaining effective teachers. Paris: OECD Publishing.

Pérez, A.I. (2010). Aprender a educar. Nuevos desafíos para la formación de docentes. Revista Interuniversitaria de Formación del Profesorado, 68(24, 2), 37-60.

Ramírez, V. (2010). El Normalismo: proyecto, procesos institucionales y actores. Revista Iberoamericana de Educación Superior, 1(2), 98-113. Recuperado de: http://ries.universia.net

Sanz, J.R., Hernando Mora, I. y Mula Benavent, J.M. (2015). La percepción del profesorado de Educación Secundaria de la Comunidad Valenciana acerca de sus Conocimientos profesionales. Estudios sobre Educación, 29, 215-234.

Shulman, L.S. (2005). Conocimiento y enseñanza: Fundamentos de la nueva reforma. Profesorado. Revista de Currículum y Formación del Profesorado, 9, 21-30.

Wei, R.C., Darling Hammond, L., Andree, A., Richardson, N. y Orphanos, S. (2009). Professional Learning in the Learning Profession. Technical Report. NSDC. Recuperado de https://learningforward.org/docs/pdf/ nsdcstudytechnicalreport2009.pdf?sfvrsn $=0$

\section{BY-NC-ND}

